\title{
Chronic Periodontitis Exacerbated by Occlusal Trauma: Report of A Case and Revision of Literature
}

\author{
Ma. Lourdes Rodriguez ${ }^{1,2 *}$, Iturralde $\mathbf{M}^{3}$, Vega $\mathrm{J}^{3}$ and Pinos $\mathrm{X}^{3}$ \\ ${ }^{1}$ Chair of Semiology and Clinical Diagnosis, University of Cuenca, Ecuador \\ ${ }^{2}$ Institute of Microbiology, Parasitology and Immunology, Faculty of Medicine, University of Buenos Aires, Argentina \\ ${ }^{3}$ Faculty of Dentistry, University of Cuenca, Ecuador
}

"Corresponding author: Ma. Lourdes Rodriguez, Professor, Chair of Semiology and Clinical Diagnosis, Faculty of Dentistry, University of Cuenca, Ecuador, Tel: 5932933554; E-mail: malourdes84@hotmail.com

Received date: March 16, 2017; Accepted date: April 4, 2017; Published date: April 11, 2017

Copyright: (C) 2017 Rodriguez L, et al. This is an open-access article distributed under the terms of the Creative Commons Attribution License, which permits unrestricted use, distribution, and reproduction in any medium, provided the original author and source are credited.

\begin{abstract}
Occlusal trauma has been associated with periodontal disease 100 years ago, but only observationally. Since the 1930s, the effect of excessive occlusal forces on the periodontium has been evaluated at the pre-clinical level. At first, studies on animal and human autopsy material showed no association between occlusal discrepancies and periodontal destruction. However, in the last 10 years new evidence has emerged that today allows us to establish a relationship between both clinical entities. The latest review on the subject published in 2015, states that at the moment there is a lack of strong evidence to assume a relation of cause/effect between periodontitis and excessive occlusal forces.
\end{abstract}

The objective of this study is to report a clinical case related to a patient with no previous medical history, with an oral diagnosis of an advanced chronic periodontitis, in which the existing occlusal trauma was recognized as a possible accelerating and/or aggravating factor. In addition there is an update of the focused theme.

Keywords: Periodontitis; Occlusal trauma; Malocclusion

\section{Introduction}

A variety of occlusal conditions have been adjudged to have a dynamic interactive impact on the periodontium, among which we can mention bruxism, mal occlusion, abfraction, etc. However the central focus has been on occlusal trauma. The International Working Group for the Classification of Periodontal Diseases and Conditions in 1999 defined occlusal trauma as an injury to the attachment apparatus or teeth as a result of excessive occlusal forces (EOF). Occlusal trauma can be divided in two general categories [1-14]:

1) Primary occlusal trauma is an injury resulting from EOF applied to a tooth or teeth with normal support [14].

2) Secondary occlusal trauma is an injury resulting from normal or excessive occlusal forces applied to a tooth or teeth with inadequate or reduced periodontal support [14].

Occlusal trauma has been associated with periodontal disease 100 years ago [2]. For example Karolyi in 1901 reported an apparent association between excessive occlusal forces and periodontal destruction [3]. In 1917 and 1926, Stillman stated that excessive occlusal forces are the primary cause of periodontal disease [4,5]. Stillman stated that occlusal forces should be controlled both to prevent and treat periodontal disease. These early reports created a background for a controversy that does not yet have international consensus on whether there is a causal relationship between occlusal discrepancies and periodontal disease, independently of the presence or absence of the factor of irritation, or whether occlusal trauma only acts as a factor that promotes the progression of plaque-induced periodontal disease.
With respect to this dilemma, in 2004 a systematic review was published in the journal Periodontology 2000 on the subject exposed and it concluded that both studies in animal and human models suggest some association between discrepancies/occlusal trauma and changes in the structures of Periodontal support. Extensive animal studies have shown that occlusal trauma has an effect on the periodontal support structures, but does not initiate the destruction of the insertion apparatus. In addition, existing research does not establish a cause and effect relationship between occlusion and periodontal disease, since there is no strong data to indicate that occlusion is a potential risk factor for mediating periodontal destruction. However, other studies have shown that the control of this risk factor can slow the progression of periodontal destruction and improve the results of the response to periodontal treatment [2].

The objective of this paper is to present a clinical case of a male patient with an oral diagnosis of generalized advanced periodontitis exacerbated by occlusal discrepancies and develop a chronological review of the topic.

\section{Case Report}

A 41-year-old male patient attended the University of Cuenca, Faculty of Dentistry, in which a general diagnosis was made and was sent to the Postgraduate Periodontics area due to an increased mobility of his teeth, accumulation of calculus, bleeding to brushing and probing, loss of insertion and halitosis (Figure 1). Regarding the general state of health, he was a healthy patient, with an asthma history background during his childhood.

After the periodontal examination, the patient was diagnosed with severe chronic generalized periodontitis exacerbated by occlusal 
Citation: Rodriguez ML, Iturralde M, Vega J, Pinos X (2017) Chronic Periodontitis Exacerbated by Occlusal Trauma: Report of A Case and

trauma. He presented mobility grade III (Miller) of dental pieces 18 ; $17 ; 16 ; 15 ; 14 ; 24 ; 25 ; 27 ; 35 ; 34 ; 32 ; 31 ; 41 ; 42 ; 43 ; 44 ; 45$. Grade II of the dental pieces $38 ; 13 ; 12 ; 11 ; 21 ; 22 ; 23$ except teeth 3.3 ; and 3.2 that presented grade I mobility; except for a retained root of the piece 27.

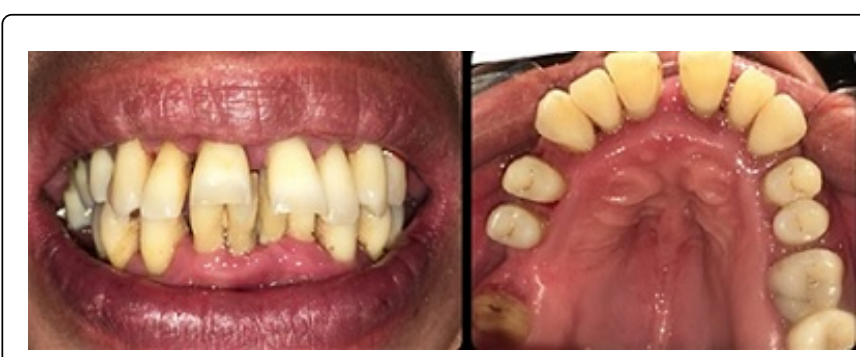

Figure 1: Intraoral photos.

An inter consult was done with the Proshetic Area to evaluate such dental pieces and make a treatment plan according to the case. The occlusal trauma could be verified (here we would have to complete in which parts the interferences were detected) and the case was evaluated in an integral way and the total extraction of the teeth was considered as therapeutic because it presented severe bone loss incompatible with any prosthetic treatment (Figure 2). The patient was submited to the Periodontal Area for the evaluation and extraction of all the dental pieces. The therapeutic scheme was designed as follows:

1. Complete blood count, hemostasis test, and glucose (Values in normal parameters).

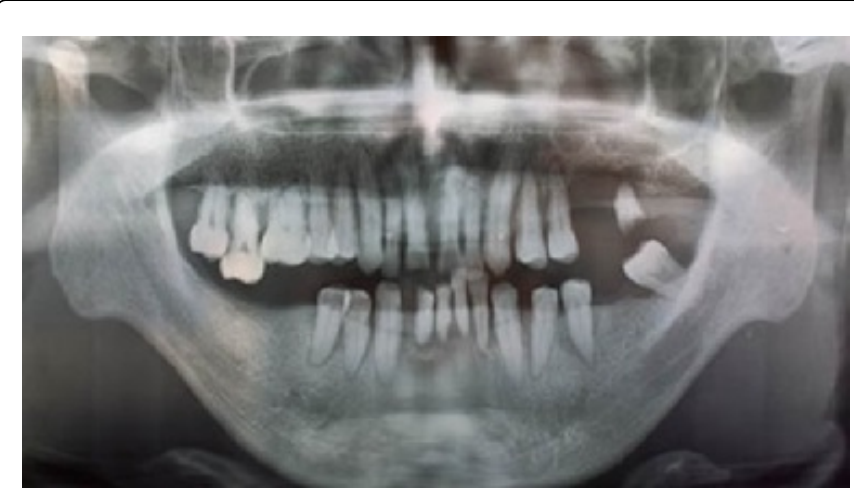

Figure 2: Panoramic x-ray; Generalized severe bone loss.

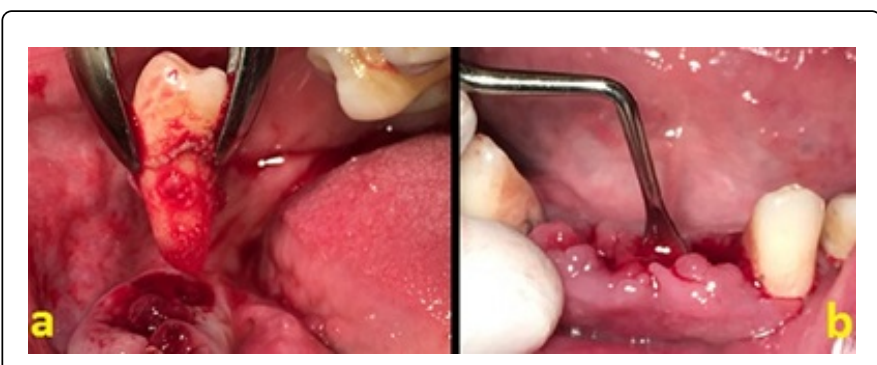

Figure 3: (a) Extraction of 4.4; (b) Curettage.

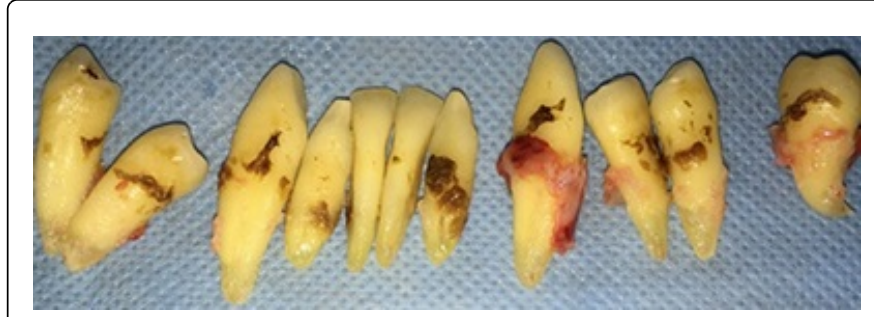

Figure 4: Inferior dental pieces.

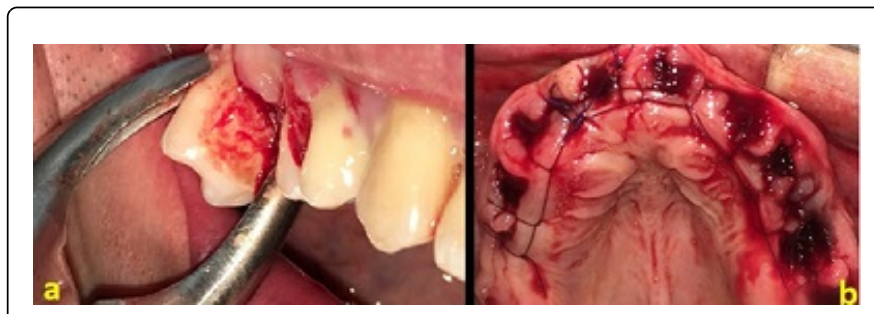

Figure 5: (a) Exodontia 1.6; (b) Continuous suture.

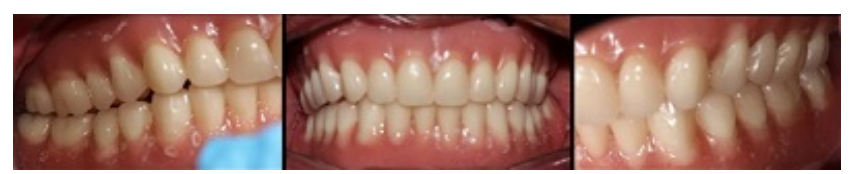

Figure 6: Upper and lower total prosthesis and tissue conditioning.

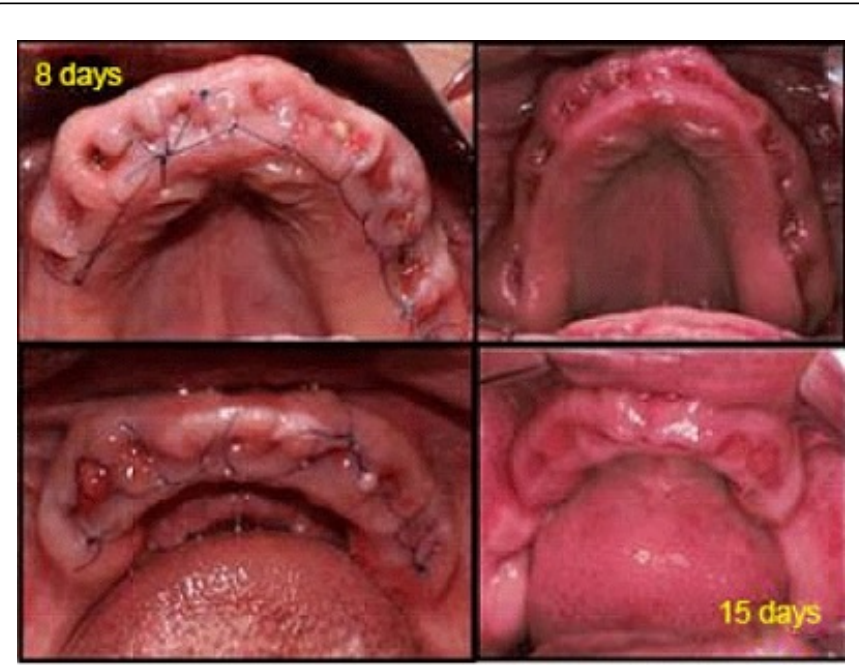

Figure 7: Postoperative 8 days and 15 days.

2. First session: the extraction of the lower teeth was performed (Figures 3 and 4) extra oral antisepsis was performed with $8 \%$ iodine, and chlorhexidine at $0.12 \%$ was used for 60 seconds for intraoral antisepsis. In each alveolus, salting and irrigation were performed with saline solution (Figure 3) it was finished with continuous suture (Figure $5 \mathrm{a}$ and $5 \mathrm{~b}$ ). 
3. Postoperative treatment: Etoricoxib $120 \mathrm{mg}$ once daily for 5 days and Amoxicillin $500 \mathrm{mg}$, one tablet every 8 hours for 7 days; cold compresses were recommended for the first 24 hours no physical exercise for a week.

4. Second session: the extraction of the upper teeth was performed (Figure 5a). For this purpose, the same protocol described above was used.

5. Within a week, the development and placement of the upper and lower prosthesis along with conditioning of the tissue was performed (Figure 6).

\section{Eight days later the removal of sutures was performed (Figure 7).}

At the moment the patient is in a waiting time to decide what will be the final treatment to be performed; he was given the option of performing total implant rehabilitation, which is compatible with the expectations he has about his treatment.

\section{Material and Method}

For updating and analysis of the topic, the PubMed and Google academic databases were accessed with the keywords "occlusal trauma" and "periodontitis"; Malocclusion and periodontitis. Only systematic reviews and preclinical studies were selected in murine models.

\section{Results and Discussion}

The role of excessive occlusal forces in the pathophysiology of periodontal disease (PD) has been disputed by several researchers, with studies emerging since the 1930 on animal models and human autopsy material [2]. The first published works on the subject did not show an obvious association between occlusal forces and periodontal disease. For example Orban and Weinmann in 1933 [6], and Weinmann in 1941 [7], using human autopsy material, evaluated the effect of excessive occlusal forces (EOF) on the periodontium. They concluded that there was no relationship between EOF and periodontal destruction, and suggested that occlusal forces have no effect on periodontal destruction. They further indicated that gingival inflammation extended through the bone support was the cause of periodontal destruction. In parallel, Glickman and collaborators [8], through their work on animal models and human autopsy material, demonstrated the existence in a periodontal initiated (by the presence of dental plaque) of an altered pathway of destruction when EOF are present. This pathway was described as a change in the orientation of the periodontal and gingival fibers under the presence of excessive occlusal forces, leading to gingival inflammation, which extends along the periodontal ligament, also producing vertical bone defects and bone loss. They concluded that EOF in the presence of plaqueassociated inflammation cause a change in the alignment of the periodontal ligaments, leading to an altered pathway of inflammation, which they called a "codestructive agent". Based on these observations, the use of occlusal adjustment was defined as part of the treatment when there was PE. Since there was no evidence that EOF started PD, occlusal adjustment to prevent periodontal disease was not recommended [8].

To carry out prospective and experimental studies in humans on the effect of occlusal forces on the progression of periodontitis is not acceptable from the ethical point of view. As a result, human studies are limited to retrospective and observational research. In this context, several results have been found, since most do not find a significant association between EOF and periodontal destruction. However, studies that included occlusal adjustment as part of periodontal treatment obtained greater insertion gain than patients who did not receive occlusal adjustment $[1,2]$.

In a retrospective report on private practice patients diagnosed with advanced periodontal disease, they were evaluated and distributed to three groups of treatment, untreated, partially treated group (basic therapy + occlusal adjustment), and fully treated group (periodontal surgery). This study differed from the previous ones in the fact that they considered each dental piece as the unit of experimentation, instead of each patient. The study evaluated the progression of periodontal destruction and periodontal evolution, for each tooth, over time, on teeth with and without occlusal discrepancies. The teeth with occlusal discrepancies had greater depth of probing and deterioration prognosis than those teeth that did not have occlusal interference. Concluding that occlusal discrepancies appear to be a risk factor that contributes to faster periodontal destruction and that treatment of occlusal interference seems to slow down periodontal destruction [2].

In a systematic review published in 2008, it was reported that studies addressing the interaction between malocclusion (MO) and periodontitis, found significant periodontal problems in subjects with MO compared to subjects without MO ( $\mathrm{p}<0.00001)$. However none of them were adjusted for confusion variables. On the other hand, two studies on the interaction between MO and gingivitis found higher levels of gingivitis in subjects with $\mathrm{MO}$ compared to subjects without MO. One of those studies was adjusted for oral hygiene. This review concluded that there is a correlation between the presence of MO and PE. Subjects with significant MO have more severe PE. This correlation may be dependent on oral health status [1].

A preclinical study was published in 2014 investigating the effects of occlusal trauma on periodontal destruction in a murine model. Sixty mice were used in the present study. Forty-eight rats immunized with lipopolysaccharide (LPS) were intra-peritoneal and divided into four groups. In the trauma $(\mathrm{T})$ group, occlusal trauma was induced by placing an excessively high metal wire on the occlusal surface of the mandibular right first molar. In the inflammation group (I), periodontal inflammation was induced by the topical application of LPS in the palatal gingival sulcus of the maxillary first maxillary molars. In the trauma + inflammation $(\mathrm{T}+\mathrm{I})$ group, both trauma and periodontal inflammation were induced simultaneously. The PBS group (control group) received only phosphate buffered saline. Another 12 non-immunized rats (the $\mathrm{n}-(\mathrm{T}+\mathrm{I})$ group) were treated as described for the $\mathrm{T}+\mathrm{I}$ group. All rats were sacrificed after 5 or 10 days; and their first upper molars with surrounding tissues were observed histopathologically Loss of insertion and osteoclasts in the alveolar bone crest were investigated histopathologically. To detect immune complexes, immune histological staining for $\mathrm{C} 1 \mathrm{qB}$ was performed. Collagen fibers were also observed using the picrosirius red polarization method. There were significant increases in insertion loss and in the number of osteoclasts in the $\mathrm{T}+\mathrm{I}$ group compared to the other groups. In addition, a generalized distribution of immune complexes was observed in the $\mathrm{T}+\mathrm{I}$ group, and the collagen fibers oriented from the root surface to the alveolar bone crest had partially disappeared in the $\mathrm{T}, \mathrm{T}+\mathrm{I}$ and $\mathrm{n}-(\mathrm{T}+\mathrm{I})$ Concluding that the damage of the collagen fibers by occlusal trauma can increase the permeability of the antigen through the tissue, and lead to the expansion of the area of immune complex formation and the acceleration of the inflammatory reaction. Therefore, periodontal tissue destruction was greater in the $\mathrm{T}+\mathrm{I}$ group than in the I group [9]. 
Citation: Rodriguez ML, Iturralde M, Vega J, Pinos X (2017) Chronic Periodontitis Exacerbated by Occlusal Trauma: Report of A Case and

Page 4 of 4

In summary, animal and human studies have suggested some association between discrepancies/occlusal trauma and changes in the structures of periodontal support $[11,12]$.

In addition, existing investigations do not establish a cause-effect relationship between occlusion and PE. Furthermore, there is no strong data to indicate that malocclusion is a potential risk factor for periodontal destruction, and that control of this risk factor may slow the progression of periodontal destruction and improve the results of the response to periodontal treatment [11].

\section{Conclusions}

After exposing the clinical case based on a chronic Periodontitis exacerbated by the existing occlusal discrepancies; and reported in the literature, we conclude that:

1. There is insufficient evidence to date to assume that there is a cause/effect association between periodontal disease and occlusal trauma.

2. Occlusal trauma is likely to act as an aggravating factor or modifier of the progression of periodontal disease, by lesioning the periodontal and gingival fibers, increasing tissue permeability, a fact that would favor the penetration of toxins and bacterial antigens, increasing this way the area of immune complex formation and therefore the inflammatory reaction at the level of the structures of periodontal support.

3. There is insufficient evidence to support occlusal adjustment to prevent periodontal disease. But if it has been scientifically proven that occlusal adjustment incorporated into periodontal treatment increases the insertion gain and slows the progression of the disease.

4. It is necessary to carry out human studies, cohorts with prospective format and clinical trials to clarify the role of occlusal trauma in the pathogenesis of periodontal disease.

\section{References}

1. Bollen A (2008) Effects of Malocclusions and Orthodontics on Periodontal Health: Evidence from a Systematic Review. Dent Edu 72: 912-918.

2. Hallmon W, Harrel S (2004) Occlusal analysis, diagnosis and management in the practice of periodontics. Periodontology 34: 151-164.

3. Karolyi M (1901) Beabachtungen über Pyorrhea alveolaris. Ost Ung Vierteljschr Zahnkeilk 17: 279-283.

4. Stillman P (1917) The management of Pyorrhea. Dent Cosmos 59: 405-414.

5. Stillman $P$ (1926) What is traumatic occlusion and how can it diagnosed and corrected. J Am Dent Assoc 12: 1330-1338.

6. Orban B, Weinmann J (1993) Signs of traumatic occlusion in average human jaws. J Dent Res 13: 216.

7. Weinmann J (1941) Progress of gingival inflammation into the supporting structures of the teeth. J Periodontol 12: 71-82.

8. Glickman I, Smulow JB (1962) Alterations in the pathway of gingival inflammation into the underlying tisssues induced by excessive occlusal forces. J Periodontol 33: 7-13.

9. Nakatsu S, Yoshinaga Y, Kuramoto A (2014) Occlusal trauma accelerates attachment loss at the onset of experimental periodontitis in rats. J Periodontal Res 49: 314-322

10. Foz A, Artese H, Horliana A (2012) Occlusal adjustment associated with periodontal therapy--a systematic review. J Dent 40:1025-1035.

11. Reinhardt R, Killeen A (2015) Do Mobility and Occlusal Trauma Impact Periodontal Longevity? Dent Clin North Am 59: 873-883.

12. Vandana K, Nadkarni R, Guddada K (2015) Comparison of various risk indicators among patients with chronic and aggresive periodontitis in davangere population. Journal of Indian Society of Periodontology 19: 429-434.

13. Hallmon W (1999) Occlusal trauma: effect and impact on the periodontium. Ann Periodontol 4: 102-107.

14. American Academy of Periodontology (1999) 1999 International Workshop for a Classification of Periodontal Disease and Conditions. Ann Periodontology 4: 102-107. 